IS CHUONG, Cheng Ming ${ }^{1}\left({ }^{1}\right.$ Dept of Path. Univ. of -5 Southern California, USA)

Development, Evolution and Engineering of Skin Appendages

In nature, different animals have different body plans and different living strategies to adapt themselves to their different ecological niche. The invention of a new organ (for example the feather) is evolution novelty. It allows animals to enter a new frontier of ecological environment. The formation of these new organs is based on the emergence of new developmental mechanisms. This so-called "evo-devo" approach represents the diverse books that can be written by the language of life, and the diversity of animals constitutes the encyclopedia of the book of life.

The feather is the centerpiece for the evolution of avian flight. The origin and evolution of feathers have long puzzled scientists. A long held view is that avian feathers evolved from elongated reptile scales, over time they progress from the plate-like scales $\rightarrow$ partially pennaceous vanes with emerging raches $\rightarrow$ bilaterally symmetric feathers $\rightarrow$ plumulaceous barbs $\rightarrow$ radially symmetric downy feathers. Two major advances in the last decade have shaken this classical view: (1) a series of fossils discoveries representing intermediate forms of feathers or feather-like appendage from the Jehol Biota of China, and (2) molecular and developmental biological experiments using chickens as a model organism. Feather forms can be modulated using retrovirus mediated gene mis-expression that mimics those found in nature today and in the evolutionary past Together the results favor an evolutionary sequence from cylindrical feather filaments splitting to form primitive barbs without barbules $\rightarrow$ radially symmetric downy feathers with plumulaceous barbs $\rightarrow$ bilaterally symmetric plumulaceous feathers $\rightarrow$ bilaterally symmetric pennaceous vanes $\rightarrow$ bilaterally asymmetric vanes. We also recognize that not all organisms with feathers are birds, and that not all skin appendages with hierarchical branches are feathers. A set of criteria is developed for true avian feathers that include 1) possessing actively proliferating cells in the proximal follicle for proximo - distal growth mode; 2) forming hierarchical branches of rachis, barbs and barbules, with barbs formed by differential cell death and can be bilaterally or radially symmetric; 3 ) having a follicle structure, with mesenchyme core during development; 4 ) when matures, consisting of epithelia without mesenchyme core and with two sides of the vane facing the previous basal and supra-basal layer respectively; and 5) having stem cells and dermal papilla in the follicle and hence the ability to molt and regenerate.

\section{Plenary Lectures (L-1 - L-2)}

L-1 HANAOKA, Fumio ${ }^{1}\left({ }^{1}\right.$ Grad. Sch. Front. Biosci., Osaka Univ. / CREST, JST / Cell. Physiol. Lab., RIKEN)

The $X P V$ (xeroderma pigmentosum variant) gene encodes a human translesion synthesis enzyme, DNA polymerase $\eta$

During the past several years a new superfamily of DNA polymerases called the Y-family has been identified. This group includes evolutionarily related proteins known to be involved in mutagenesis. Several of these enzymes support replicative bypass of damaged bases that arrest high-fidelity, highly processive DNA polymerases involved in DNA replication. This process (translesion DNA synthesis) lies at the center of mutagenesis. Human DNA polymerase $\eta(\mathrm{Pol} \eta$ ) was found to be a gene product responsible for an autosomal recessive disease, namely the variant form of xeroderma pigmentosum (XP-V), characterized by a high incidence of sunlight induced skin cancer. Pol $\eta$ belongs to the Yfamily of DNA polymerases. At first sight it may appear paradoxical that the inactivation of a low fidelity DNA polymerase, such as Pol $\eta$ in XP-V cells, renders cells hypermutable to UV light. This paradox is best understood on the basis of the capacity of Pol $\eta$ to read efficiently through the major UV lesion, a TT cyclobutane dimer, by inserting two adenines across the dimer thus restoring the correct sequence. We have found that the fidelity of translesion synthesis by human Pol $\eta$ relies not only on the ability of this enzyme to incorporate the correct nucleotide opposite a lesion, but also on its ability to elongate only DNA chains that have a correctly incorporated nucleotide opposite a lesion. Nevertheless, the fidelity and processivity of DNA synthesis by Pol $\eta$ on undamaged templates are extremely low, suggesting that Pol $\eta$ activity must be restricted to damaged sites on DNA. We have recently shown that Pol $\eta$ binds template/primer DNAs regardless of the presence of cyclobutane dimers. Rather, enhanced binding to template/primer DNAs containing TT dimers is only observed when 3'-end of the primer is an adenosine residue situated opposite the lesion. When two nucleotides have been incorporated into the primer beyond the TT dimer position, the Pol $\eta$-template/primer DNA complex is destabilized, allowing DNA synthesis by DNA polymerase $\alpha$ or $\delta$ to resume. Our study provides mechanistic explanations for polymerase switching at TT dimer sites.

L-2 NAGATA, Shigekazu ${ }^{1}\left({ }^{1}\right.$ Lab. of Genet., Integrated Biol. Lab., Grad. Sch. of Frontier Biosci., Osaka Univ. and Dept. of Genet., Osaka Univ. Med. Sch.)

Molecular Mechanism of Apoptosis and its Failure

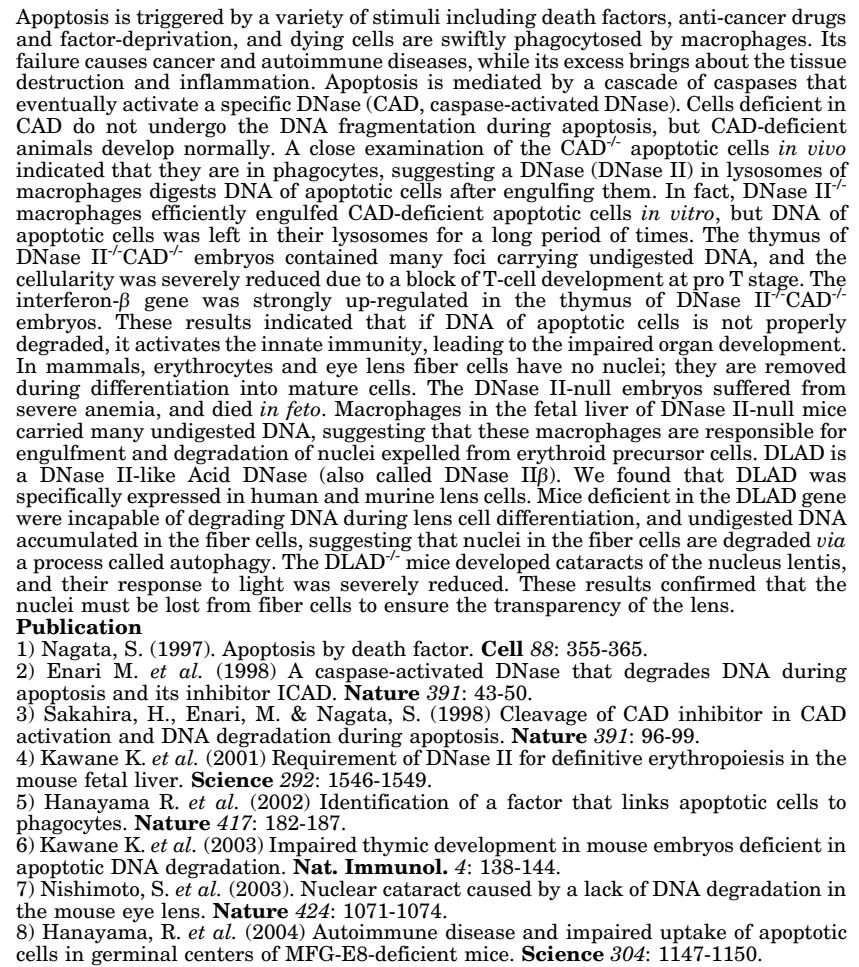

and factor-deprivation, a variety of stimuli including death factors, anti-cancer drugs destruction and inflammation. Apoptosis is mediated by a cascade of caspases that eventually activate a specific DNase (CAD, caspase-activated DNase). Cells deficient in CAD do not undergo the DNA fragmentation during apoptosis, but CAD-deficient inimals develop normally. A close examination of the CAD apoptotic cells in vivo macroph thes macrophages efficiently engulfed CAD-deficient apoptotic cells in vitro, but DNA of apoptotic cells was left in their lysosomes for a long period of times. The thymus of The interferon- $\beta$ gene was strongly up-regulated in the thymus of DNase $\mathrm{II}^{-} \mathrm{CAD}^{-\mu}$ embryos. These results indicated that if DNA of apoptotic cells is not properly In mammals, erythrocytes and eye lens fiber cells have no nuclei; they are removed during differentiation into mature cells. The DNase II-null embryos suffered from severe anemia, and died in feto. Macrophages in the fetal liver of DNase II-null mice carried many undigested DNA, suggesting that these macrophages are responsible for a DNase II-like Acid DNase (also called DNase II $\beta$ ). We found that DLAD was specifically expressed in human and murine lens cells. Mice deficient in the DLAD gene were incapable of degrading DNA during lens cell differentiation, and undigested DNA accumulated in the fiber cells, suggesting that nuclei in the fiber cells are degraded via a process called autophagy. The DLAD mice developed cataracts of the nucleus lentis, nuclei must be lost from fiber cells to ensure the transparency of the lens.

Publication

2) Enari M. et al. (1998) A caspase-activated DNase that degrades DNA during apoptosis and its inhibitor ICAD. Nature 391: 43-50.

3) Sakahira, H., Enari, M. \& Nagata, S. (1998) Cleavage of CAD inhibitor in CAD activation and DNA degradation during apoptosis. Nature 391: 96-99.

4) Kawane K et al. (2001) Requirement of DNase II for definitive erythropoiesis in the mouse fetal liver. Science 292: 1546-1549.

5) Hanayama $\mathrm{R}$ et al. (2002) Identification of a factor that links apoptotic cells to phagocytes. Nature 417: 182-187.

apoptotic DNA degradation. Nat. Immunol 4: 138-144. 7) Nishimoto, S. et al. (2003). Nuclear cataract caused by a lack of DNA degradation in 8) Hanayama, R. et al. (2004) Autoimmune disease and impaired uptake of apoptotic
cells in germinal centers of MFG-E8-deficient mice. Science 304: 1147-1150. 